\title{
Flow past a Sphere
}

\section{Kern E. Kenyon}

4632 North Lane, Del Mar, USA

Correspondence to: Kern E. Kenyon, kernken@aol.com Keywords: Flow past a Sphere

Received: March 5, $2019 \quad$ Accepted: March 24, $2019 \quad$ Published: March 27, 2019

Copyright $\odot 2019$ by author and Scientific Research Publishing Inc.

This work is licensed under the Creative Commons Attribution International License (CC BY 4.0).

http://creativecommons.org/licenses/by/4.0/

\section{(c) (i) Open Access}

\section{ABSTRACT}

A new theoretical framework is applied to the steady fluid flow past a solid smooth sphere. Bernoulli's law along a streamline is combined with the cross-stream force balance: centrifugal force on the curved flow equals a pressure gradient. When compared with the standard potential theory for flow past a sphere in a text book, the prospect of a major discrepancy is found. Whereas the decay rate of the velocity perturbation away from the sphere goes as the inverse cube of the distance in the text book, the decay rate computed here is in all likelihood very different, and it depends on an unknown constant function, the radius of curvature of the streamlines versus distance from the sphere. When that function is supplied either from another theory or from detailed observations (probably streak photographs), then the new approach can be solved completely. In any case, accurate measurements of flow rates at different positions with respect to the solid are badly needed.

\section{INTRODUCTION}

Leonardo da Vinci wrote down in a notebook his observation of the flow past a rock in a stream: the flow is fastest at the sides of a covered rock rather than above it [1]. Unfortunately he did not write about the rate at which the fastest flow decayed outward from the rock toward the normal mean stream velocity. How far apart should two rocks in a stream be in order that their respective perturbed velocities do not interfere with each other? To this day, I have not seen observations relevant to answering this question. It is not that the subject is so unimportant either, because it is tied up with understanding how birds and planes fly.

A theory exists, but to my knowledge no observations have ever been compared with it. Potential flow is the name of the theory, which has been applied to flow past a circular cylinder in many text books as well as flow past a sphere in one text that I have [2]. It would not seem to be very difficult to obtain experimental evidence, such as carefully made streak photographs. After all, Leonardo used bird seed and other small floating objects to obtain enlightening visual images.

As presented in the text books, the potential flow theory is incomplete because the pressure field sur- 
rounding the sold body is never shown, only the velocity field. Not just that limitation, but within itself the theory looks to be inconsistent or contradictory. For example, the die off rate for the velocity perturbation extending radially out from a circular cylinder varies as the inverse square of the distance, whereas for the sphere the rate varies as the inverse cube of the distance. That makes no sense physically.

\section{NEW APPROACH}

There is another theoretical framework available, which has some advantages over the classical method, and it has already been applied to the steady flow past a circular cylinder [3]. This new approach can also just as easily be applied to flow past a sphere. One of the advantages is that the die off rate for velocity perturbations is the same for both the sphere and the cylinder, which is a more reasonable outcome. Another advantage is that the pressure perturbation is readily calculated and presented.

Consider a steady uniform flow encountering a fixed smooth solid sphere and limit the mean speed so that no eddies form at the back face of the sphere. Gravity and friction are not included in the model. In a plane parallel to the mean flow that slices through the middle of the sphere the streamlines separate in going around the circular cross-section of the sphere. Let the z-axis point up at the top of the circle.

Bernoulli's law applies to all streamlines going over the top (and bottom) of the solid circle.

$$
p=\text { const }-\frac{1}{2} \rho V^{2}
$$

where $p$ is the pressure, $V$ is the speed of flow in the vicinity of the solid body and $\rho$ is the constant fluid density. Far away from the sphere $V$ trans forms into the uniform flow speed $U$. For simplicity the constant in (1) is taken the same for all streamlines.

Fluid following a curving path above the sphere's circular cross-section experiences an upward centrifugal force. When the flow is steady there must be an equal but opposite force, and in this situation it can only be a pressure gradient. Therefore the cross-stream force balance is

$$
\frac{\mathrm{d} p}{\mathrm{~d} z}=\frac{\rho V^{2}}{R}
$$

In (2), $R(z)$ is the variable radius of curvature of the streamlines, which at the top of the sphericalcross-section becomes the constant radius of the circle, $R_{o}$.

Equations (1) and (2) are two equations in the two unknowns, velocity and pressure. Each equation by itself is nonlinear. But between the two equations one of the variables can be eliminated, and it does not matter which, because the result is a linear equation in each case. For example, the velocity equation is

$$
\frac{\mathrm{d} V}{\mathrm{~d} z}=-\frac{V}{R}
$$

which is linear although the unknown constant $R(z)$ varies with $z$. Equations (1) and (2) are exactly the same for the circular cross-section of the sphere as they are for the circular cylinder [2]. As a consequence, by skipping steps the solution can be written down immediately

$$
V=V_{0}\left(e^{f(z)}-1\right)+U
$$

where $V_{0}$ is a constant that can be determined by conserving mass between two vertical cross-sections, one on top of the sphere and the other far away from it, and

$$
f(z)=\int_{0}^{z} \frac{\mathrm{d} Z}{R(Z)}
$$

\section{DISCUSSION}

Equation (4) is by no means a complete solution of the problem for the velocity perturbation, but it 
contains strong hints that the potential flow method has flaws that may eventually render its usefulness questionable. If Equation (5) could be integrated, either algebraically or numerically, that would be a good start. Detailed measurements of the radius of curvature function $R(z)$ are needed to evaluate (5) numerically. While waiting for that to happen a couple of algebraic representations of $R(z)$ have been tried out to see what the die off rate looks like for the velocity perturbation. So far nothing approximating the inverse square or inverse cube law of the distance has emerged.

As mentioned above the pressure equation is quickly obtained from (1) and (2) by eliminating the velocity to give

$$
\frac{\mathrm{d} p}{\mathrm{~d} z}=-\frac{2 p}{R}
$$

which is similar to (3) with $p$ replacing $V$ except for the factor of 2 in the numerator of the RHS. That factor of 2 is critical in the sense that it means the pressure perturbation is more tightly bound to the solid body (cylinder or sphere) than the velocity perturbation is no matter what the radius of curvature function turns out to be.

Finally comes a thought experiment, within the same general theoretical framework, but strictly speaking outside the bounds of Equation (2) in the above model. Drop the sphere and greatly expand the (horizontal) scale such that the centrifugal force on the RHS of Equation (2) is replaced by the Coriolis force. Then when the pressure is substituted out between (1) and (2), a linear equation in the horizontal velocity shear results

$$
\frac{\mathrm{d} V}{\mathrm{~d} s}=-f
$$

where $f$ is the Coriolis parameter, which locally is a constant (sign/hemisphere left unspecified), and measures horizontal distance normal to the mean flow direction. This combination of Bernoulli's law with the geostrophic relation has not occurred in print before as far as I am aware. One reason for such an omission may be that until recently Bernoulli's law has never been associated with large-scale phenomena, such as certain weather systems over the North Pacific [4].

\section{CONCLUSION}

Based on an apparent internal inconsistency in the theory and a complete lack of any comparisons between theory and measurements, it is predicted that the applications of potential flows past circular cylinders and spheres will not lead to increased understanding of these phenomena. Although incomplete at this time, a new theoretical approach is under development as outlined above that may be more helpful in this regard in the future.

\section{CONFLICTS OF INTEREST}

The author declares no conflicts of interest regarding the publication of this paper.

\section{REFERENCES}

1. MacCurdy, E., Ed. (1948) The Notebooks of Leonardo Da Vinci. Volume 2, Jonathan Cape, London, 90.

2. Faber, T.E. (1995) Fluid Dynamics for Physicists. Cambridge University Press, Cambridge, 133. https://doi.org/10.1017/CBO9780511806735

3. Kenyon, K.E. (2013) Flow past a Cylinder. Journal of Scientific Theory and Methods, 2013, 211-222.

4. Kenyon, K.E. (2018) Bernoulli Weather or Not? Natural Science, 10, 178-181. 